\section{Diagnosis of brain death}

SIR,-I find myself in a strange position with regard to the current brain death debate. I am a doctor and also chairman of the Association of Community Health Councils. From where I am I think that I really can see both sides.

I would like to suggest that the BMA (or the colleges) approach the BBC and apply to do an Open Door programme under its community access scheme. No doubt the application could be backed up by the suggestion that the programme could be made available to medical schools, postgraduate centres, and patient and community groups as part of continuing education about transplants. This basis for action would avoid the problems of editorial freedom and allow the profession to produce its version of a balanced programme. I would obviously press the case for the involvement of patients and community interests in the making of the programme.

Of course, these programmes are not transmitted at peak times but at least the resources of the BBC would be seen to be available. It might even be that some part of the BBC current affairs machine chose to feature parts of the programme at a peak time-subject, of course, to editorial judgments, news value, and so on.

Editorial freedom is one of those things, like clinical freedom, that we all have to be prepared to die for; the pity at the moment is that it is only those with chronic renal failure who are having actually to make the sacrifice. I offer my admittedly naive suggestion as a way in which both the profession and the media could work together and climb up out of this mess rather than wait for either to climb down.

\section{R K GRIFFITHS}

Health Services Research Centre, University Department of Social Medicine

Birmingham B15 2T

\section{Toxic shock and tampons}

SIR,-We wish to draw attention to a case of toxic shock syndrome in Auckland, New Zealand, with reference to your leading article (1 November, $\mathrm{p}$ 1161)

On 22 July 1980 a 21-year-old New Zealand woman was admitted to hospital at the end of a normal period, during which she uninterruptedly used a brand of tampons bought and manufactured in New Zealand. She was extremely unwell with a four-day history of diarrhoea and one to two days of vomiting, rigors, myalgia, and sore throat. She had facial oedema, conjunctivitis, a widespread diffuse erythematous rash, and pharyngitis. She was shocked and her blood pressure was $75 / 40 \mathrm{~mm} \mathrm{Hg}$. Vaginal examination was normal apart from a slight vaginal discharge and there was no evidence of pelvic inflammatory disease. Penicillin-resistant Staphylococcus aureus was cultured from the vaginal discharge but no pathogens were cultured from other specimens including blood cultures. She was resuscitated, given intravenous antibiotics, and recovered. Desquamation of the rash began on the fifth hospital day and continued for two weeks. Staph aureus was not cultured from her vagina after antibiotic therapy finished.

Two months later she was readmitted with a similar though less severe illness at the end of a period, during which she had used the same brand of tampons. This time she had a profuse vaginal discharge but again a striking absence of objective signs of pelvic inflammatory disease. Staph aureus was again cultured from the discharge but attempts to culture it from elsewhere in her body were not successful. She took cloxacillin and recovered, and the organism was eliminated. She has remained well. Staph aureus was not cultured from the unused boxed tampons.

Toxic shock syndrome, as might be expected, is not seen only in the United States, and tampons manufactured elsewhere may thus be causally associated. This woman had a recurrence despite initial elimination of vaginal Staph aureus. Despite widespread medical and lay publicity in New Zealand generated by this patient, we are unaware of others. This case is being reported in detail in the New Zealand Medical Fournal.

S IEA

Auckland Hospital,
Auckland 1, New Zealand

R B ElLIS-PEGLER

SIR,-We were surprised to read in your leading article "Toxic shock and tampons" (1 November, $\mathrm{p} \mathrm{1161)}$ that "A significant association has been shown between the use of tampons .... and the development of the toxic shock syndrome. In one study Staph aureus was isolated from 43 of 44 vaginal cultures taken from women with the toxic shock syndrome before antimicrobial treatment was started, whereas it was isolated from the vagina of only four of 55 control women. ..." While this suggests an association between Staph aureus and the syndrome it neither examines nor provides evidence regarding the role of tampons.

One of us chanced to be in the United States recently when this syndrome was the subject of massive publicity. As there are some 80 million menstruating women in the United States, $70 \%$ of whom it is estimated use tampons, this publicity seemed a contribution to the creation of disproportionate anxiety, ill health, and probably futile activity.

JAMES MCCORMICK ShaNe AllWRIGHT University Department of Community Health, Dublin 2

* * Our leading article stated that " $95 \%$ of all reported cases of toxic shock syndrome in women have occurred during or soon after a menstrual period" and analysis showed a substantial excess of users of one particular brand of tampon. How much plainer could an association be ?-ED, $B M \mathcal{F}$.

Staphylococcal septicaemia after insertion of an intrauterine contraceptive device

SIR,-Your recent leading article on toxic shock and tampons (1 November, $p$ 1161) prompts me to report a case of pelvic infection and staphylococcal septicaemia eight days after the insertion of a Lippes Loop. Pelvic infection is a recognised complication of intrauterine contraceptive devices (IUCD) ${ }^{\mathbf{1}}$; but although there have been two reports of endocarditis occurring in susceptible patients following the insertion of an IUCD ${ }^{23}$ septicaemia is rare.

A previously healthy 31-year-old married woman had a Lippes Loop IUCD fitted at a family planning clinic. Three days later she developed sweating, vomiting, confusion, and cough and during the following 48 hours became disorientated with hallucinations. She was referred to hospital with suspected encephalitis and on admission was febrile $\left(38.8^{\circ} \mathrm{C}\right)$ and stuporose but responded to simple commands. Blood pressure was $95 / 60 \mathrm{~mm}$ $\mathrm{Hg}$ but there were no other abnormal signs. Haemoglobin was $12.2 \mathrm{~g} / \mathrm{dl}$, white blood count $4.0 \times 10^{9} / 1$ ( $80 \%$ neutrophils), erythrocyte sedimentation rate $70 \mathrm{~mm}$ in the first hour; cerebrospinal fluid normal. Chest $x$-ray examination revealed patchy consolidation in the upper lobes of both lungs and an electroencephalogram showed bilateral non-specific abnormality.

Three blood cultures taken on admission yielded penicillin-resistant Staphylococcus aureus. She was treated with high-dose intravenous cloxacillin and 24 hours after starting the antibiotic had improved markedly and the IUCD was removed. Culture from the coil and also from a high vaginal swab yielded Staph aureus with a similar antibiogram to that of the organism cultured from the blood. Subsequent recovery was uneventful, although repeat chest $x$-ray examination showed small abscess cavities in the upper lobes of both lungs. The patient was discharged four weeks after admission and serial chest radiographs have confirmed complete resolution of the pneumonia and abscesses.

There is little doubt that this patient's staphylococcal septicaemia with lung abscess formation and encephalopathy originated in the genital tract. The patient was both toxic and shocked but was different from patients with the recently described toxic shock syndrome in that her blood culture was positive for Staph aureus. The case provides another example of the importance of this organism as a cause of infection associated with the insertion of foreign bodies into or through the vagina.

A M GedDES

\section{Department of Communicable and Tropical Diseases,}

Birmingham B9 5ST

1 Anonymous. Br Med f 1976;ii:717-8.

3 de Swiet. M, Ramsay ID, Rees GM. Br Med f 1975;iii:76-7.

\section{Fulminant Streptococcus pyogenes}

\section{infection}

SIR,-I read with interest your "Lesson of the Week" (22 November, p 1412). We had a patient in February 1978, who did not present a classical picture.

A 46-year-old woman was admitted to the surgical ward with severe intractable pain in her right hip. She had had a "flu-like" illness with a sore throat for one week, as had her daughter. She had received no antibiotics. The pain had become progressively more severe over the previous 24 hours. She was dehydrated and confused with a pyrexia of $37 \cdot 6^{\circ} \mathrm{C}$. There was no history of trauma but she had chronic lumbar disc disease. Over the next few hours she developed an area of bruising, oedema, and skin desquamation over her right hip and buttock; this spread rapidly over her thigh. It was not a true cellulitis, just a raw bleeding area where the skin had sloughed off. The area was very cold to touch. She had three sets of blood cultures and throat and skin swabs taken. Benzylpenicillin, 5MU six hourly, and gentamicin were started intravenously, as necrotising fasciitis was suspected. She became shocked 24 hours after admission (10 hours after starting antibiotics) and was resuscitated with plasma. Twelve hours later the lesions had spread down to her right knee, extending as far as her lumbar region and across the midline. She then had a cardiac arrest and all attempts to resuscitate her failed.

Streptococcus pyogenes ( $\beta$-haemolytic streptococcus, Lancefield group A) was later isolated from her blood cultures and throat and skin swabs. At necropsy she had a grossly oedematous 
right thigh with discoloration and bruising. The superficial epithelium had sloughed off. Her lungs, spleen, and liver were congested. Str pyogenes was isolated from her skin and subcutaneous tissue, but not from her heart blood or spleen. Her right hip showed no underlying abnormality.

This is another example of a patient with Str pyogenes infection who did not have a classical presentation. This case also emphasises the fulminating nature of the infection, as she died despite having been on appropriate high doses of benzylpenicillin for 20 hours.

Penelope J Redding

Department of Bacteriology and Immunology, Glasgow G11 6NT

\section{Comparison of neonatal management} methods for very low birthweight babies

SIR,-Dr E S Steiner and others (8 November, p 1237) have offered a very succinct paper examining the outcome of very low birthweight infants who did not require "special or intensive" care. I am unable to agree with their conclusions and am saddened by their hostility towards intensive perinatal care.

Clearly none of their infants required intensive care intervention and those that did died. Thus a population of "well babies" remained, of whom only $52 \%$ were normal at school age. Surely these results are disastrous and must indicate that more intensive care facilities are required at this unit.

A more useful comparison would be a similar population at University College Hospital or Hammersmith Hospital-that is, very low birthweight infants who did not require intensive therapy after birth and who were managed by minimal handling only. The figures quoted from these other units included babies ventilated for hyaline membrane disease. I would be very interested to learn whether Dr Pamela Davies at Hammersmith Hospital has been able to differentiate non-ventilated very low birthweight infants in her follow-up studies.

G J REYNOLDS

Institute of Child Health, Alder Hey Children's Hospital,
Liverpool L12 2AP

* * We sent this letter to Dr Davies, who replies below.-ED, $B M \mathcal{~}$.

SIR,-Thank you for giving me the opportunity to reply to Dr Reynolds's query about the outcome for non-ventilated very low birthweight infants. In the 15-year (1961-75) survey of infants weighing $\leqslant 1500 \mathrm{~g}$ at their birth in Hammersmith Hospital ${ }^{1}$ to which Dr E S Steiner and his colleagues refer (8 November, p 1237) 148 of the total of 357 were ventilated, and $94 \%$ of them died. The fate of the 209 non-ventilated infants was as follows: $70(33.5 \%)$ died in the neonatal period, 5 $(2 \cdot 4 \%)$ later, $5(2 \cdot 4 \%)$ were untraced, 17 $(8.1 \%)$ had major and $20(9.6 \%)$ minor handicap as defined, ${ }^{1}$ and $92(44.0 \%)$ were apparently normal. The figures for major and minor handicap reported by Dr Steiner and his colleagues were $5.5 \%$ and $12.3 \%$ respectively.

\section{P A Davies}

\section{Hammersmith Hospital}

1 Jones RAK, Cummins M, Davies PA. Lancet 1979; i:1332-5.

\section{Intrinsic hazard of breech presentation}

SIR,-The management of breech delivery continues to be widely debated. Caesarean section is increasingly used to deliver mature breech babies. Some obstetricians advocate abdominal delivery for those between $\mathbf{3 2}$ and 37 weeks while others include very small prematures-that is, those between 26 and 32 weeks. Presumably the operation referred to has always been the lower-segment one.

Mr John P Calvert (15 November, p 1319) now suggests that ill, small breech babies should be delivered by classical section because the fetal head might be delivered more quickly for resuscitation. Surely the risks of such a policy must far outweigh the advantages. The uterine wound is often larger with the classical procedure and when the placenta is anterior the blood loss at operation will often demand transfusion Paralytic ileus, generalised peritonitis, and occasionally acute small bowel obstruction are complications to be reckoned with in the puerperium. ${ }^{1}$ But the over-riding argument against the use of classical section for small compromised breech is that the scar can and does rupture in any subsequent pregnancy with the risk of maternal death and a fetal mortality rate of approximately $75 \%{ }^{2}$ The results quoted by $\mathrm{Dr}$ Calvert suggest that if a breech baby has to be delivered by section then a colleague skilled in resuscitation must be in the theatre. Perhaps the operation should be performed under epidural block using all methods to prevent the supine hypotensive syndrome. Certainly all obstetric anaesthetic skills are essential. The abdomen should be opened by a mid-line incision an the fetus delivered through an adequate transverse incision in the lower segment.

Perinatal statistics have become too important. It is wiser to deliver a young teenager of a 35-week breech vaginally using an epidural block than to seriously prejudice her obstetric career let alone her life by performing a classical section. Similarly, if the cord prolapses during the labour of a smal premature breech baby it is often sensible not to operate. A stillborn 30 -week fetus with the uterus left intact is preferable in a young woman than to leave her with a scarred upper segment and a brain-damaged baby.

New Cross Hospital

Alan M SMITH

Wolverhampton

${ }^{1}$ Myerscough, P. Munro Kerr's operative obstetrics. 8th edn. London: Balliere Tindall, 1977:511. Dewhurst CJ. Fournal of Obstetrics and Gynaecology of the British Empire 1957;64:113-8.

\section{Kidney biopsy}

SIR,-The juxtaposition of two letters (4 October, p 945) containing similar statistics, but supporting two different techniques for kidney localisation prior to biopsy, will not help those trying to decide which method they should use. Our letter from Nottingham was not written with the knowledge that it would be adjacent to the vigorous advocacy for urographic localisation put forward in the Bristol letter. It is clear that both techniques are satisfactory when used by experienced staff, and a choice between the two cannot be made on the basis that one is more likely to be successful, or is less prone to complications.

The merits of ultrasound are in part logistic; it is quicker to establish the position of the kidney than with urography. The technique is especially useful iwhen it is uncertain if urography will demonstrate the kidney. It provides the operator with the distance of the kidney capsule from the skin. In our opinion the role of biopsy with urography will be for the few failures under ultrasound, when the depth of the kidney will already be known.

It seems certain that very occasional severe renal haemorrhage will occur with all methods. It is surprising that neither the original article nor the correspondence comments on the treatment of this complication by selective embolisation of the bleeding kidney. We hesitated to be advocates of this technique with only two severe haemorrhages to report. The first followed a "blind" biopsy in acute renal failure before the era of selective embolisation and nephrectomy was performed after 10 days of bleeding. In the second patient, with chronic renal failure and before the use of ultrasound, a selective embolisation stopped prolonged and severe bleeding with no detectable impairment of renal function. It is important that those performing renal biopsy should be aware of the technique, as it appears much preferable to nephrectomy. Its availability has reduced to some extent the caution with which we recommend renal biopsy.

MARTIN S KNAPP

R P BURDEN

D H ROSE

Peter Davies

City Hospital

Nottingham NG5 1PD

\section{Renal aneurysms in rejected renal} transplants

SIR,-The finding of multiple small intrarenal aneurysms by Dr J F. Walker and others (8 November, p 1251) prompted us to review the renal arteriograms performed in our unit during the past seven years with a view to determining the incidence of such aneurysms, both in general and in our own transplant series.

In this period 918 renal arterial studies were performed with selective renal arteriography, flush aortography, or a combination of both techniques, depending on the clinical indication for the study. In some examinations (for example, transplanted kidneys) just one kidney was examined but in most others both kidneys. Indications for the procedure included suspected tumour (262 kidneys), suspected rejection or arterial stenosis in a renal transplant (136 kidneys), suspected polyarteritis nodosa (102 kidneys), the assessment of prospective renal donors (162 kidneys), and miscellaneous pathology (hypertension, abnormal urogram, haematuria, etc, 256 kidneys). In the total series of 918 organs studied, multiple small intraparenchymal aneurysms were demonstrated in 24 kidneys (14 patients); four of these kidneys (four patients) were rejected transplants and the other 20 (10 patients) were in cases of polyarteritis nodosa Aneurysms were also seen in association with hypertension, stenosis, and atherosclerosis and following renal biopsy, renal surgery, or renal trauma. These aneurysms all affected the large intrarenal arteries or the extrarenal arteries, and were usually solitary; their appearance was quite distinct from the multiple small lesions of polyarteritis or transplant rejection. Bizarre aneurysmal vessels were also seen in the pathological circulation of renal tumours; the lesions in these cases were confined to the region of the tumour.

It has been noted that aneurysms were demonstrated in four out of 136 transplanted kidneys examined arteriographically ( $3 \%$ ). A furthe 